\title{
Das neue Reservierungssystem WLB-Riserva
}

Alle öffentlichen Einrichtungen, darunter Bibliotheken, leisten ihren Beitrag zur Eindämmung der Corona-Pandemie. Auch die Württembergische Landesbibliothek stand 2020 vor der Herausforderung, ein Konzept für die Abstandsregelungen, Besucherzahlbeschränkungen und Kontaktpersonennachverfolgung in kürzester Zeit zu entwickeln und umzusetzen. So wurde in der WLB innerhalb weniger Wochen das Online-Reservierungssystem WLB-Riserva durch einen ehemaligen IT-Mitarbeiter in Zusammenarbeit mit den Kollegen*innen der Information und der IT-Abteilung entwickelt.

Wo vor der Pandemie die Türen der WLB für alle Besucher*innen ohne Einschränkungen offen standen, hatten die Corona-Regeln Auswirkungen auf das Platzangebot und auf die Nutzungsmöglichkeiten. So konnten von den 378 Arbeitsplätzen nur 193 angeboten werden. Diese müssen über WLB-Riserva reserviert werden. Eine Besonderheit, die das Reservierungssystem von anderen unterscheidet, ist der Buchungsprozess. Die Arbeitsplatzzuweisung geschieht in zwei Schritten: Zunächst wird zum gewünschten Zeitfenster ein Arbeitsbereich reserviert; erst durch die Buchung - das "Einchecken" - vor Ort wird dann ein bestimmter Arbeitsplatz zugeteilt. Das ermöglicht die bestmögliche und flexible Vergabe der Plätze, so dass auch auf kurzfristige Änderungen reagiert werden kann.

\section{Persönliche Kontofunktionen}

Das Reservierungssystem ist über unsere Homepage verlinkt. Die Anmeldung erfolgt über den Bibliotheksaccount. In WLB-Riserva stehen neben den Arbeitsplätzen zum Lernen und Lesen verschiedene Ressourcen wie zum Beispiel die Buchscanner, ePflicht-PCs oder der barrierefreie Arbeitsplatz zur Auswahl. Darüber hinaus kann ein Arbeitsplatz im Bereich des bevorzugten Fachgebietes ausgewählt werden. Die Reservierung garantiert zunächst nur die Verfügbarkeit eines Platzes zum gewünschten Zeitpunkt, wenn möglich auch im gewünschten Fachbereich.

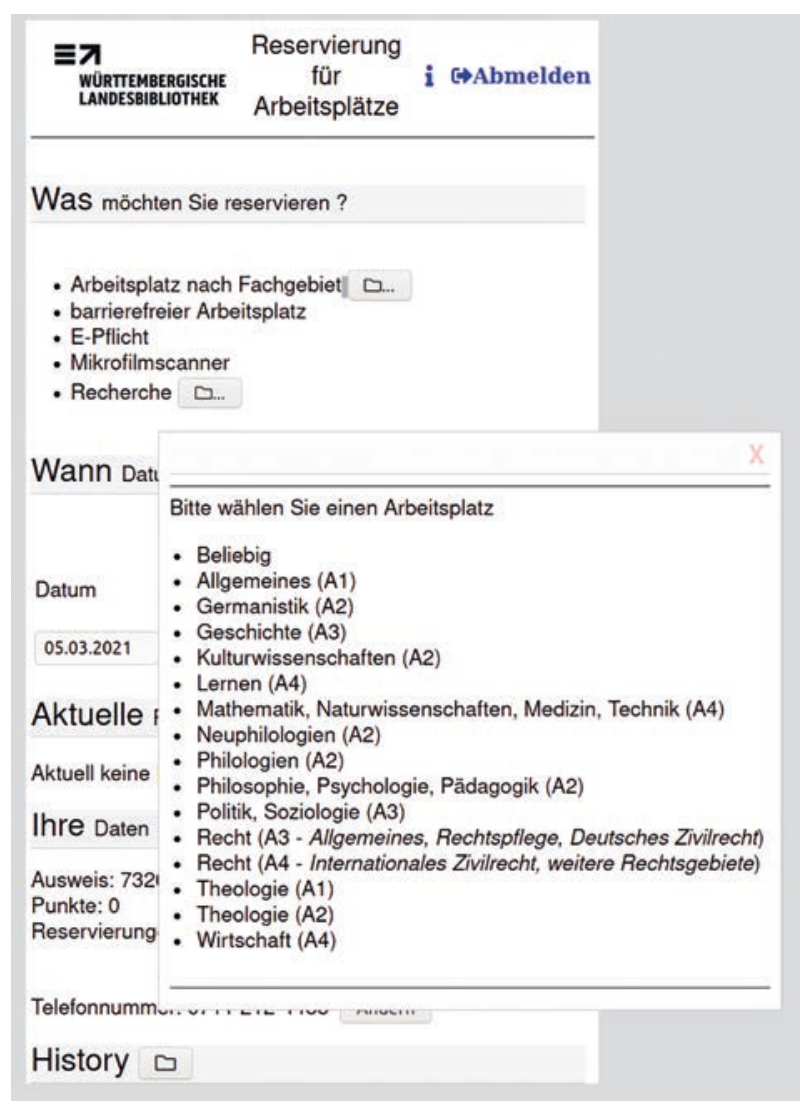

Abb. 1: Auszug Platzreservierung in WLB-Riserva

Nach dem Login sind im Konto des Nutzers sowohl die getätigten Reservierungen, die bereits erfolgten Buchungen sowie der aktuelle Reservierungspunktestand ersichtlich. Pro 15 Minuten Arbeitszeit wird dabei ein Reservierungspunkt vergeben. Die Höhe des Punktestandes hat Auswirkungen darauf, wie weit Reservierungen in der Zukunft getätigt werden können. Je höher der Punktestand, desto weniger Tage kann im Voraus reserviert werden. Dies ermöglicht eine gerechte Verteilung der Arbeitsplätze und verhindert, dass wenige Bibliotheksbenutzer*innen die Ressourcen "fluten". Es besteht auch die Möglichkeit, getätigte Reservierungen zu stornieren. Die Gutschrift der Punkte ist in diesem Fall gewährleistet. 


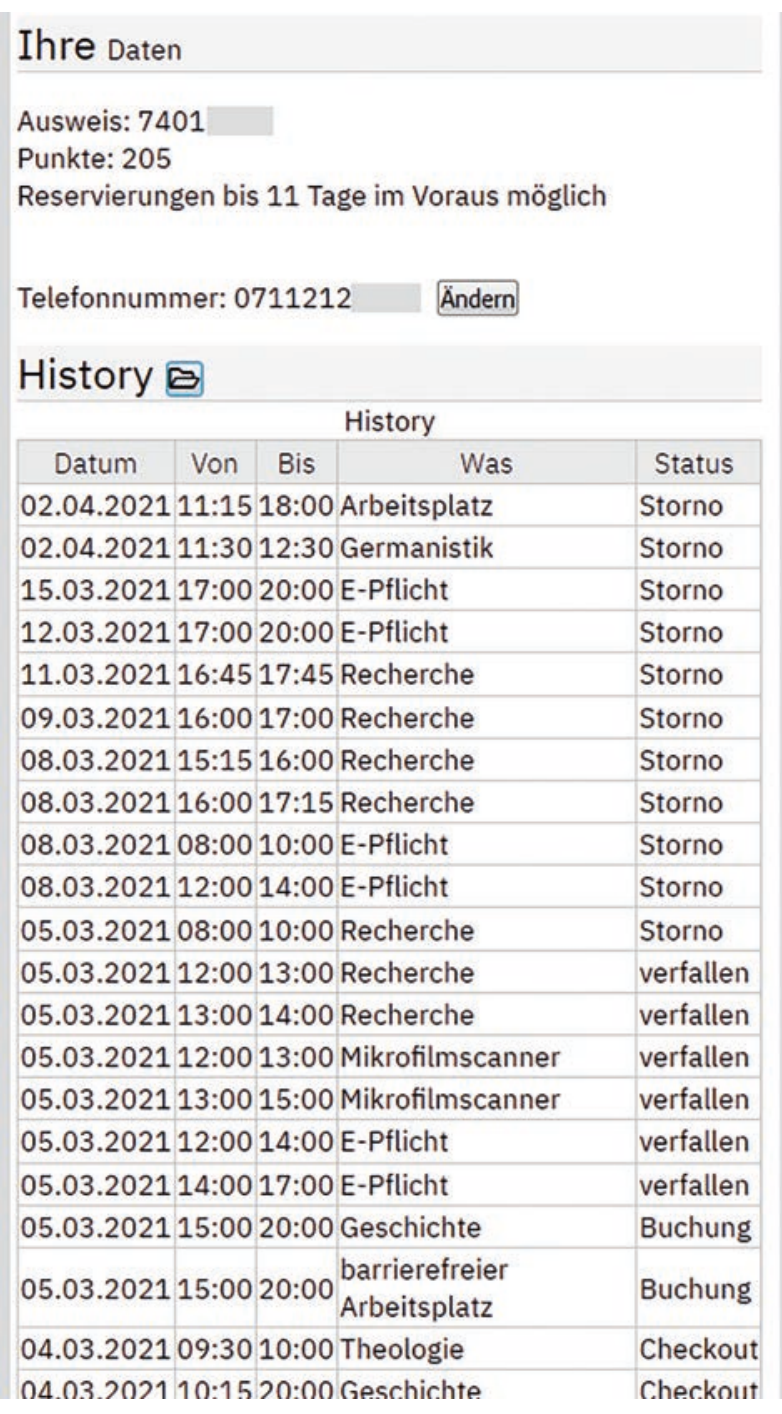

Abb. 2: WLB-Riserva Kontoaktivität

\section{Check-in}

Eine konkrete Platzzuweisung erfolgt beim „Einchecken“ an einem Buchungsterminal, die im Empfangs- und Eingangsbereich aufgestellt sind. Die Reservierung wird über den Benutzerausweis kontaktlos abgerufen. Als Buchungsbestätigung wird ein Bon mit der Tischnummer, dem Zeitfenster und den Bibliotheksausweisangaben ausgedruckt, der auf Nachfrage den WLB-Mitarbeiter*innen vorgezeigt werden muss. Alternativ kann die Buchung mit dem Smartphone erfolgen, wenn dieses im WLB-WLAN eingebucht ist. Buchungen sind bis zu 15 Minuten vor dem Reservierungsstartzeitpunkt möglich, um den Arbeitsplatz rechtzeitig erreichen zu können. Jedoch verfällt die Reservierung 30 Minuten nach dem Beginn der Reservierungszeit. Dieser Arbeitsbereich wird dann im System als frei markiert. Wird der Platz früher verlassen, so können über die
Aus-Check-Funktion die verbleibenden Reservierungspunkte gutgeschrieben werden.

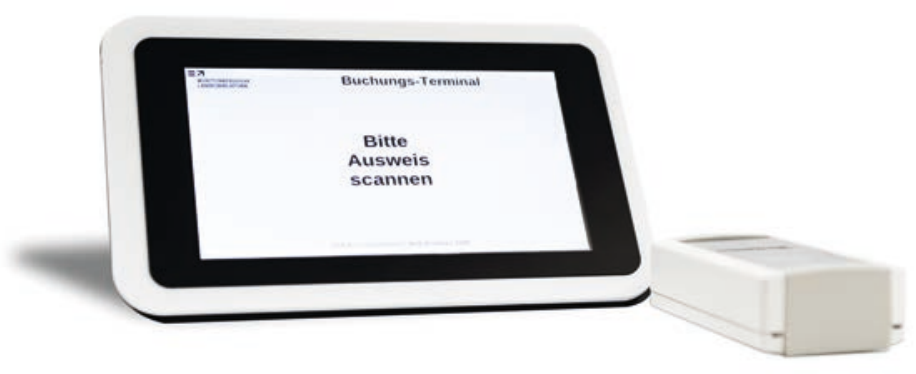

Abb. 3: Buchungsterminal im Empfangsbereich

\section{Konfiguration und Erweiterungen}

Das Reservierungssystem ist so aufgebaut, dass es leicht administrierbar und optimierbar ist. Basierend auf WLB-Riserva entwickelte die IT-Abteilung weitere Module, beispielsweise die automatische Benachrichtigung über die annullierten Reservierungen, eine Kontaktpersonennachverfolgung bei Corona-Fällen und ein Modul zur Ermittlung der Benutzungsstatistiken. Auch nachträgliche Anpassungen wie zum Beispiel Standortwechsel der Arbeitsbereiche, die im neuen Erweiterungsbau immer wieder stattfinden, sind schnell in das Reservierungssystem einzupflegen.

Im Falle der nicht vorhersehbaren, pandemiebedingten Schließung der WLB für den Publikumsverkehr oder bei zeitweisen Teilsperrungen von Arbeitsplätzen besteht die Möglichkeit, die Bibliotheksbenutzer*innen automatisch mit der hinterlegten E-Mail-Adresse über die annullierten Reservierungen zu benachrichtigen.

Für die Nachverfolgung der Infektionsketten besteht die Möglichkeit, die Buchungen einer durch das Gesundheitsamt als infiziert gemeldeten Person zu markieren und automatisch eine Liste der Kontaktpersonen auszugeben. Somit können die Gesundheitsbehörden die betroffenen Benutzer*innen zeitnah informieren, falls diese sich in der Nähe des Quellfalls über längere Zeit aufgehalten haben. Das händische Pflegen von Listen vor Ort entfällt dadurch und die Schlangenbildung kann vermieden werden. 
Zurzeit wird die WLB-Riserva-Statistikseite weiterentwickelt. Durch das Statistikmodul wird es den WLB-Mitarbeiter*innen ermöglicht, sich besser auf die Benutzerbedürfnisse einzustellen und sich auf den zu erwartenden Nutzungsbetrieb vorzubereiten. Dazu werden die Statistiken zu Auslastung, Verfallsquote, Fächerpräferenzen, Benutzungszeiten, Arbeitsplatzpräferenzen, Randzeitennutzung, etc. automatisch ermittelt. Mitarbeiter*innen können die folgenden Aspekte abfragen: Wie ausgelastet ist aktuell die Bibliothek (auch pro Stockwerk), wieviel Besucher werden heute erwartet, in welchem Umfang werden die Reservierungen tatsächlich gebucht und wieviel davon sind verfallen, welche Tageszeiten sind beliebt und welche Fächer sind besonders nachgefragt. Das Statistikmodul kann um weitere Auswerteaspekte ergänzt werden. Diese Auswertungen geben der WLB eine belastbare Grundlage, um die Nutzungsparameter sinnvoll justieren zu können.

\section{Fazit}

WLB-Riserva hat für einen stabilen und reibungsloseren Benutzungsbetrieb in der von Corona geprägten Zeit gesorgt und ist für weitere Anforderungen anpassungsfähig und erweiterbar. Der zweistufige Buchungsvorgang erforderte jedoch aus Sicht der Bibliotheksbenutzer*innen eine gewisse "Eingewöhnung".

\section{Stephan Abele / Aysoltan Gravina}

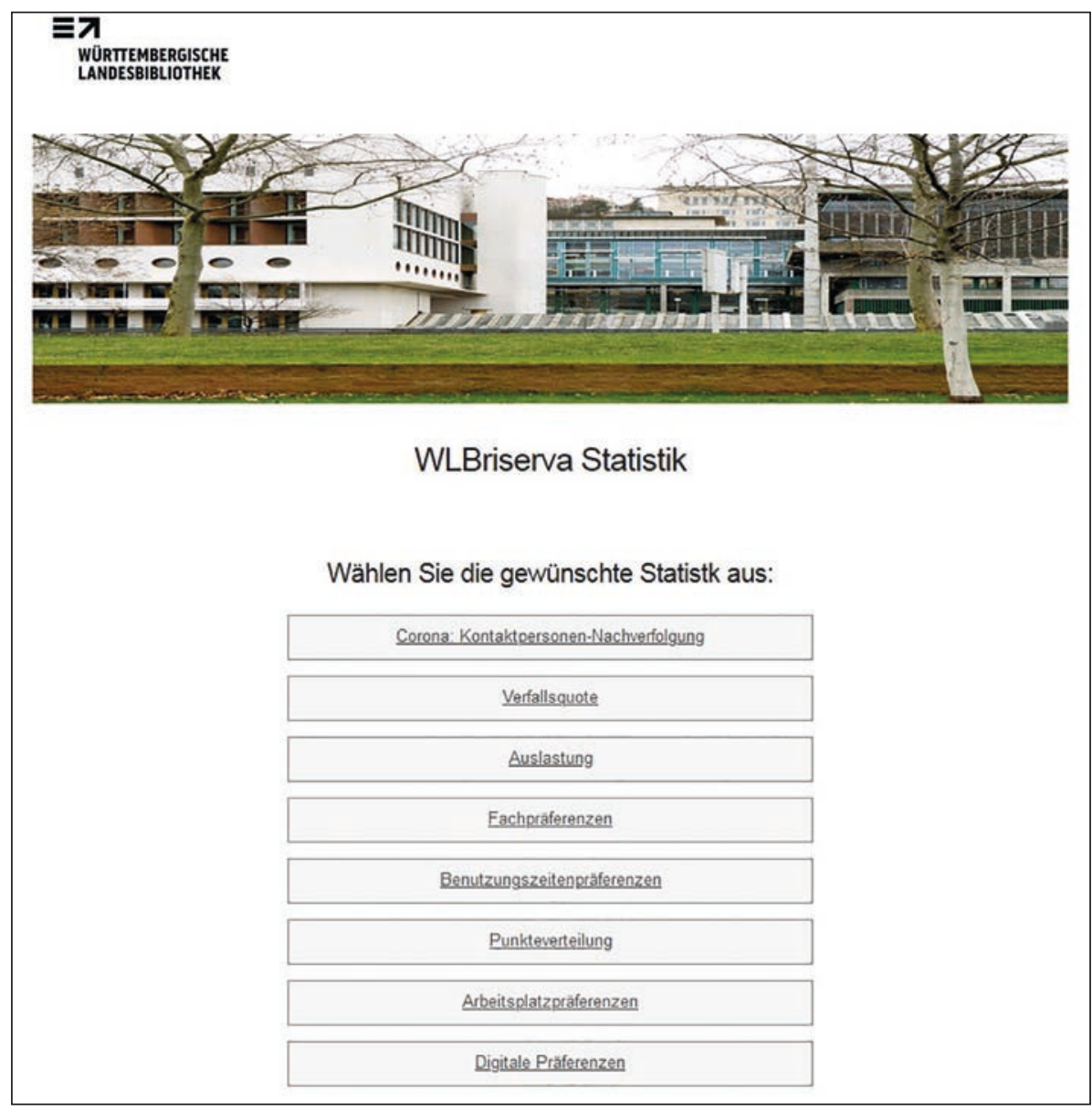

Abb. 4: Startseite des WLB-Riserva-Statistikmoduls 